\title{
Serious disease outbreaks caused by viruses transmitted by Aedes aegypti in Brazil
}

\author{
Luiz Tadeu Moraes Figueiredo ${ }^{[1]}$
}

\begin{abstract}
[1]. Centro de Pesquisa em Virologia, Faculdade de Medicina de Ribeirão Preto, Universidade de São Paulo, Ribeirão Preto, São Paulo, Brasil.
\end{abstract}
Currently, a tragic combination of disease outbreaks caused by viruses transmitted by the mosquito Aedes aegypti occurs in Brazil. This African insect infests most of the urban areas of Brazil, as it is completely adapted to the urban conditions, and it is responsible for the transmission of viruses belonging to the genus Flavivirus, family Flaviviridae [dengue viruses (DENV 1-4) and Zika virus (ZIKV)], and genus Alphavirus, family Togaviridae [Chikungunya virus (CHIKV)]. Aedes aegypti has been introduced into Brazil many times during centuries.

The arrival of yellow fever virus (YFV) and its vector, A.aegypti, in Brazil is most likely related to the slave trade and commodities trade, and it was brought by ships carrying infected individuals. YFV caused urban outbreaks in the XVII, XIX, and $\mathrm{XX}$ centuries, in the Brazilian coastal and coffee-farming areas. However, at some point, YFV jumped from the cycle involving humans-A. Aegypti to a sylvatic cycle involving Haemagogus mosquitoes of the forest canopy trees, and this virus is maintained as a primate zoonosis in the Amazon forest, Pantanal, and Central Plateau of Brazil. However, YFV causes epizootic outbreak waves through the river gallery forests, which leads to small outbreaks among humans in the highly populated regions of South and Southeast Regions of Brazil. Fortunately, YFV has not caused urban outbreaks to date, which are transmitted by $A$. aegypti, due to an efficient control of the virus, based on the excellent 17D vaccine. For safety reasons, and for the efficient protection of urban areas against YFV, $90 \%$ vaccination coverage should be achieved among the population ${ }^{(1)}$.

Dengue outbreaks have occurred in Brazil since XIX century, but during the last 30 years, four DENV serotypes have been introduced in the most populated areas of the country, DENV-1 in 1985, DENV-2 in 1990, DENV-3 in 2000, and DENV-4 in 2008 . These viruses have spread and were probably reintroduced many times, producing subsequent large outbreaks that affect millions, cause severe disease in tens of thousands people, and

Corresponding author: Dr. Luiz Tadeu Moraes Figueiredo.

e-mail:1tmfigue@fmrp.usp.br

Received 24 May 2016

Accepted 3 June 2016 lead to thousands of deaths. DENV serotype alterations increase the number of secondary infections and of the severe forms of disease. The circulation of DENV belonging to serotypes and genotypes associated with the severe forms of disease aggravates this situation further. DENV-1 has been the predominant serotype in 2016 outbreak. As a consequence of the endemic nature of this disease, this virus has caused severe forms of disease among children, leading to a large number of deaths ${ }^{(2)}$.

Two CHIKV genotypes were introduced in Brazil, most likely in 2013, from the Caribbean and Asia. In September 2014, an outbreak in Feira de Santana, Bahia, was the first documented outbreak of CHIKV. Asian genotype CHIKV spread through Northeast (Bahia), Midwest, and Southeast (São Paulo) Regions of Brazil ${ }^{(3)}$. In 2015, 20662 suspected cases of Chikungunya fever were reported in the outbreaks that occurred in the States of Amazonas, Ceará, Goiás, Maranhão, Minas Gerais, Pará, Paraná, Pernambuco, Rio de Janeiro, Rio Grande do Sul, and São Paulo. Moreover, three fatal cases were reported, all in elderly $(85,83$, and 75 years old) with chronic diseases. CHIKV infection is spreading even further, and in 2016 alone, 3,768 suspected cases of Chikungunya fever have been reported before February ${ }^{(4)}$. CHIKV infection leads to a febrile illness affecting joints, and many patients show signs of acute arthritis. Ankles, wrists, and small joints of the hands are the most affected. However, larger joints, such as knees, shoulders, and spine, may also be involved. A bigger issue is that, as the studies in Asia showed, in more than $2 \%$ of individuals, these symptoms can become chronic. The joint disease is associated with the persistent presence of virus, which presents as viral osteomyelitis. The affected joints also show signs of inflammatory processes, and high levels of cytokines and chemokines are produced by synovial tissue cells. The presence of the virus and the accompanying inflammation can lead to the destruction of articular cartilage and bone ${ }^{(5)}$. Large outbreaks of CHIKV may increase the number of individuals with chronic physical disabilities.

Another Alphavirus, Mayaro virus (MAYV), is endemic in the Amazon and the Central Plateau of Brazil, leading to the outbreaks of acute febrile illness affecting joints ${ }^{(6)}$. However, the circulation of this virus, which causes zoonotic disease of primates, is increasing. In the last three years, MAYV-caused 
outbreaks have been reported in Goiás State. MAYV may represent a serious threat especially if it adapts to an urban cycle involving $A$. aegypti, and gains the ability to cause large outbreaks in the most populated areas of the country ${ }^{(7)}$.

In Brazilian cities infested by Aedes aegypti, the introduction of ZIKV led to the worsening of complex Brazilian epidemiological scenario involving arboviruses. ZIKV human infections were initially reported in February 2015 in Bahia State. Very quickly, the virus spread toward other Northeastern States, Rio Grande do Norte, Alagoas, Maranhão, and to the North as well ${ }^{(4)}$. In 2016, ZIKV outbreaks have been reported in all Brazilian regions except in the South Region. It is believed that the Asian strain of ZIKV, very likely originating in Polynesia, has been introduced into Brazil by travelers, in 2013 or 2014. The virus was initially thought to cause benign acute febrile illness accompanied with rash and conjunctivitis. However, the reports of severe forms of Zika fever surprised Brazilian and world public. Guillain-Barré syndrome is a serious neurological disease, in which autoantibodies damage the axons of motor neurons as a consequence of a viral infection. The axon lesions disrupt the neurotransmission, leading to muscle paralysis ${ }^{(8)}$. Four states in the Northeast Region reported an increase of more than $100 \%$ in Guillain-Barré syndrome incidence, which has pointed to the connection of these cases with ZIKV infection. In 2015, 1,708 cases of Guillain-Barré syndrome were reported in Brazil and the numbers were particularly higher in the Northeast ${ }^{(4)}$. In 2016, Guillain-Barré syndrome cases have been described in most of the large Brazilian cities. However, despite a growing body of evidence, World Health Organization (WHO) considers that it is too early to attribute the increase in the incidence of Guillain-Barré syndrome to ZIKV infections.

Starting from September 2015, an increase in the incidence of fetal microcephaly was observed in the Northeast Region of Brazil, and it was soon associated with a maternal infection with $\mathrm{ZIKV}^{(9)}$. Until March 2016, 6,776 suspected cases of microcephaly have been investigated across the country. ZIKV infection was confirmed in 108 of these children ${ }^{(4)}$. The congenital disease caused by ZIKV, with microcephaly as its most severe manifestation, has led to the emergence of worldwide concern, and it resulted in an international research effort to obtain information on the different aspects of the disease, including clinical presentation of the disease in the mother and the fetus, disease incidence, physiopathology, and the development of diagnostic methods and vaccines against ZIKV. Since then, physicians and scientists from Brazil and other countries have shown that ZIKV causes a severe congenital disease by infecting the central nervous system of the fetus in all phases of pregnancy. ZIKV is a neurotropic virus, and high viral loads have been found in the nervous tissues of the stillborn children of mothers infected with $\mathrm{ZIKV}^{(10)}$. Information has been collected as fast as possible to help the management of patients, provide the guidance to women in childbearing age and pregnancy, and to control the outbreaks.

Nevertheless, further studies are necessary to identify the real dimension of ZIKV and CHIKV outbreaks, and to show the connection between the clearly severe clinical manifestations and the causative viruses. Further steps that should be performed are as follows: a) to study large groups of pregnant women infected with ZIKV and the newborns, and in the case of CHIKV infection, to study patients with severe or chronic arthritis; b) to develop simple, rapid, and reliable laboratory tests for a simultaneous diagnosis, which would allow the discrimination between the infections caused by DENV 1-4, CHIKV, and ZIKV. This discrimination should be possible in both the acute viremia and in the convalescent phase of the diseases. These laboratory tests should be used in all clinical laboratories across the country, including those in the hospitals, and not only in the laboratories of the Ministry of Health; c) to develop antiviral drugs for the treatment of the acute phase of dengue, Chikungunya, and Zika diseases. Passive immunization with specific neutralizing antibodies should be provided and drugs which are able to control inflammatory cytokine storm in severe cases (in hemorrhagic dengue cases, in pregnant women infected with ZIKV, and patients with severe or chronic arthritis caused by CHIKV).

Finally, the development of highly effective vaccines against these viruses, similar to the vaccine against yellow fever, represents an urgent task. Vaccines against DENV, ZIKV, and CHIKV may be used at once, as a mixture, or sequentially, in order to immunize the entire population.

\section{Conflicts of Interest}

The authors declare that there is no conflict of interest.

\section{REFERENCES}

1. Monath TP, Vasconcelos PF. Yellow fever. J ClinVirol 2015; 64:160-173.

2. Figueiredo LTM. Dengue in Brazil: 1999-2009. Dengue Bull (WHO) 2010; 34:6-12.

3. Figueiredo MLG, Figueiredo LTM. Emerging alphaviruses in the Americas: Chikungunya and Mayaro. Rev Soc Bras Med Trop 2014; 47:677-683.

4. Brazilian Ministry of Health. Dengue, Chikungunya, Zika, Syndrome of Guillain- Barré. March 2016.

5. Chen W, Foo S-S, Sims NA, Herrero LJ, Walsh NC, Mahalingam S. Arthritogenic alphaviruses: new insights into arthritis and bone pathology. Trends Microbiol 2015; 23:35-43.

6. Mourão MPG, Mota BR, Souza GS, Bastos MS, Gimaque JBL, Galusso ES, et al. Mayaro fever in Manaus, Brazil, 2007-2008. Vect Born Zoon Dis 2012; 12:42-46.

7. Long KC, Ziegler SA, Thangamani S, Hausser NL, Kochel TJ, Higgs S, et al. Experimental transmission of Mayaro Virus by Aedes aegypti. Am J Trop Med Hyg 2011; 85:750-757.

8. Brasil P, Sequeira PC, Freitas AD, Zogbi HE, Calvet GA, de Souza $\mathrm{RV}$, et al. Guillain-Barré syndrome associated with Zika virus infection. Lancet 2016; 387:1482.

9. Butler D. Zikavirus: Brazil's surge in small-headed babies questioned by report. Nature 2016; 530:13-14.

10. Brasil P, Pereira Jr JP, Raja Gabaglia C, Damasceno L, Wakimoto M, Ribeiro Nogueira RM, et al. Zikavirus infection in pregnant women in Rio de Janeiro - preliminary report. N Engl J Med 2016; Mar 4 [Epub ahead of print]. PMID: 26943629. 